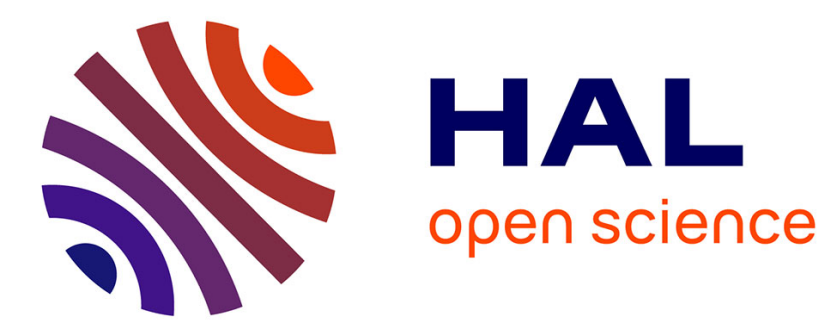

\title{
The Equation of State of the Hard-Disk Fluid Revisited
}

\author{
J. Ramon Solana, Angel Mulero, Isidro Cachadina
}

\section{To cite this version:}

J. Ramon Solana, Angel Mulero, Isidro Cachadina. The Equation of State of the Hard-Disk Fluid Revisited. Molecular Physics, 2009, 107 (14), pp.1457-1465. 10.1080/00268970902942250 . hal00513289

\section{HAL Id: hal-00513289 \\ https://hal.science/hal-00513289}

Submitted on 1 Sep 2010

HAL is a multi-disciplinary open access archive for the deposit and dissemination of scientific research documents, whether they are published or not. The documents may come from teaching and research institutions in France or abroad, or from public or private research centers.
L'archive ouverte pluridisciplinaire HAL, est destinée au dépôt et à la diffusion de documents scientifiques de niveau recherche, publiés ou non, émanant des établissements d'enseignement et de recherche français ou étrangers, des laboratoires publics ou privés. 


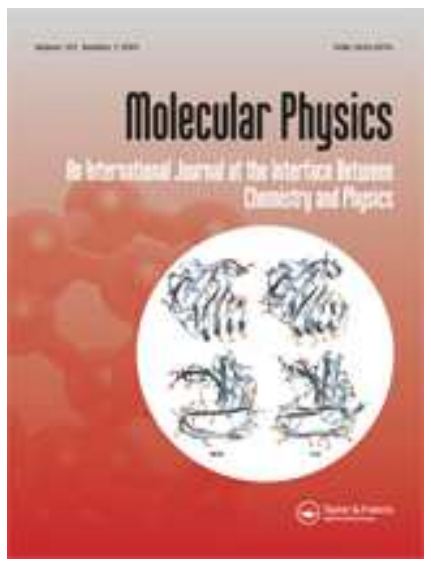

\section{The Equation of State of the Hard-Disk Fluid Revisited}

\begin{tabular}{|r|l|}
\hline Journal: & Molecular Physics \\
\hline Manuscript ID: & TMPH-2008-0418.R1 \\
\hline Manuscript Type: & Full Paper \\
\hline Date Submitted by the & 01-Apr-2009 \\
\hline Complete List of Authors: & $\begin{array}{l}\text { Solana, J. Ramon; Universidad de Cantabria, Fisica Aplicada } \\
\text { Mulero, Angel; Universidad de Extremadura, Departamento de } \\
\text { Fisica Aplicada } \\
\text { Cachadina, Isidro; Universidad de Extremadura, Departamento de } \\
\text { Fisica Aplicada }\end{array}$ \\
\hline Keywords: & equation of state, hard-disk fluid, virial coefficients \\
\hline Note: The following files were submitted by the author for peer review, but cannot be converted \\
to PDF. You must view these files (e.g. movies) online.
\end{tabular}

\section{scholarONE" \\ Manuscript Central}




\title{
The Equation of State of the Hard-Disk Fluid
} Revisited

\author{
A. Mulero ${ }^{a, 1}$, I. Cachadiña ${ }^{a, 2}$, and J. R. Solana ${ }^{b *}$ \\ ${ }^{a}$ Departamento de Física Aplicada, Universidad de Extremadura, \\ 06071 Badajoz, Spain \\ ${ }^{b}$ Departamento de Física Aplicada, Universidad de Cantabria, \\ 39005 Santander, Spain
}

March 31, 2009

\footnotetext{
*Corresponding author. Email: ramon.solana@unican.es. Phone: +34 942 201447. Fax: +34 942201407.

${ }^{1}$ Email: mulero@unex.es

${ }^{2}$ Email: icacha@unex.es
} 


\begin{abstract}
Some points about the search for analytical expressions for the equation of state of the hard-disk fluid are discussed in the light of the most recent advances in the field. New and accurate equations of state for this fluid are proposed.
\end{abstract}

Keywords: equation of state; hard-disk fluid; virial coefficients 


\section{Introduction}

As is well known, the hard-disk (HD) system is defined by an interaction potential that considers only the repulsive forces between molecules [1]. The simplicity of this model allows one to calculate its thermodynamic properties by obtaining analytical solutions of some theories or by performing computer simulations. Nevertheless, the results given by the various methods can be analytically and numerically different. In particular, the equation of state (EOS) of a system is perhaps its most important thermodynamic relationship. Unfortunately, there is no exact theoretical solution for the EOS of this two-dimensional system. As a consequence, different equations of state for the hard-disk fluid there have been proposed for many years, and the situation continues nowadays, as from time to time new equations of state are proposed for this fluid $[2,3]$.

There are different methods to propose a new EOS. Thus, sometimes the proposed equations of state have a theoretical basis. More frequently the proposed equations of state are approximants based on the known virial coefficients. Often, the reported equations are empirical or semiempirical in character. In any case, and given the great number of proposals, some efforts have been made in order to study their applicability and accuracy $[2,3,4,5]$. In particular, Mulero et al. [3] have recently collected more than thirty expressions for the HD EOS proposed in the literature. The main features or some interesting aspects of each of the expressions were reported and, for an important number of them, some studies to test their accuracy to reproduce the compressibility factor of the system by comparing with available computer simulation data were described in detail.

Almost simultaneously a new proposal was published [6], which was not included in the above mentioned study. Unfortunately, the four new expressions proposed by Khanpour and Parsafar, were only compared with the one of Santos et al. [4], and therefore some other more recent proposals $[2,7,8,9]$ were not taken into account.

In this paper, the EOS for HDs is revisited and the comparisons performed by Mulero et al. [3] and by Khanpour and Parsafar [6] are actualized. To do this, the recent com- 
puter simulation data obtained by Kolafa and Rottner [9] are taken as a reference, and then results for the compressibility factor are compared in different density ranges. In addition, new accurate expressions are proposed. The viewpoint adopted throughout this paper is that any new proposed equation of state should meet at least one of the following conditions: 1) It is more accurate than any other previously proposed equation of state in reproducing the available simulation data for the same property. 2) The new equation of state is more accurate than any other in reproducing the know virial coefficients for this kind of fluid and at the same time provides reasonable agreement with the simulation data for the equation of state. 3) The proposed equation has a sounded theoretical basis and provides reasonable accuracy as compared with the simulation data for the equation of state and with the known virial coefficients. 4) The new equation is simple and provides good agreement with simulation data and virial coefficients and considering together simplicity and accuracy is advantageous with respect to other equations of state previously reported. Obviously, other equations of state not fulfilling any of these conditions might be proposed, and in fact they are, but their usefulness is doubtful.

In the next sections we will analyze the state of the art with regard to these points before presenting the new proposals, but first we will summarize the present knowledge about simulation data for the equation of state and exact or accurate numerical calculations of the virial coefficients.

\section{Virial coefficients for the hard-disk fluid and simulation data for the equation of state}

As is well known, the EOS of a pure substance is usually given in terms of the density and temperature dependence of the compressibility factor $Z=p / \rho k T$, where $\rho=N / V$ is the number density. For HDs the compressibility factor is temperature-independent and its density dependence is commonly expressed using the so-called packing fraction, $\eta=\pi \rho \sigma^{2} / 4 \equiv \pi \rho^{*} / 4$, where $\sigma$ is the diameter of the disks [3] and $\rho^{*}=\rho \sigma^{2}$ is the reduced 
density. The maximum value of $\eta$ is the so-called closest-packing fraction, $\eta_{c}=\frac{\pi \sqrt{3}}{6}$, which correspond to a reduced density, $\rho_{c}^{*}=2 / \sqrt{3} \approx 1.1547$.

The problem of obtaining an EOS for any fluid could be solved apparently if one could determine the coefficients in the infinite virial series expansion of the compressibility factor in powers of the density, i.e.

$$
Z=1+\sum_{n=2}^{\infty} B_{n} \rho^{n-1}
$$

$B_{n}$ being the $n$th virial virial coefficient. Although the convergence of the virial series is not guaranteed for all state points, the fact is that the more virial coefficients are known, the more accurate is the equation of state obtained from the virial expansion. In any case, only the first few virial coefficients of this expansion are known at best and, since the virial series converges slowly, frequent use is made of several types of approximants in order to accelerate the convergence of the series (some of these approximants are described in the following sections). In particular, for the hard-disk system, the first ten virial coefficients are known, although important statistical uncertainties must be taken into account beyond the fourth $[8,9,10]$. Their values are as follows $[8]$

$$
\begin{aligned}
& B_{2}=(1 / 2) \pi \sigma^{2} \quad ; \quad B_{3} / B_{2}^{2}=4 / 3-\sqrt{3} / \pi \quad ; \quad B_{4} / B_{2}^{3}=2-(9 / 2) \sqrt{3} / \pi+10 / \pi^{2} \quad ; \\
& B_{5} / B_{2}^{4}=0.33355604(1) \quad ; \quad B_{6} / B_{2}^{5}=0.1988425(42) \quad ; \quad B_{7} / B_{2}^{6}=0.1148728(43) \quad ; \\
& B_{8} / B_{2}^{7}=0.0649930(34) \quad ; \quad B_{9} / B_{2}^{8}=0.0362193(35) \quad ; \quad B_{10} / B_{2}^{9}=0.0199537(80)
\end{aligned}
$$

In the preceding expressions, the numbers between parenthesis indicate the uncertainty in the last decimal places. Estimations of the virial coefficients from the eleventh to the sixteenth have been performed recently [9, 10], obtained on the basis of approximants constructed from the lower order virial coefficients and simulation data. 
With respect to computer simulations, many have been carried out in order to obtain values of the compressibility factor for different density ranges, and a summary of them can be seen in Ref. [3]. Until recently, the most accurate simulation data available for the equation of state of the hard-disk fluid were those reported by Erpenbeck and Luban [13] for the range $1 / 30 \leq \rho / \rho_{c} \leq 1 / 1.4$. Nevertheless, simulation data for the fluid in the range $0.40 \leq \rho^{*} \leq 0.90$ have been recently reported by Kolafa and Rottner [9]. At intermediate densities very good agreement with Erpenbeck and Luban's data is found. Due to the advanced techniques used, the Kolafa and Rottner values are considered as the most accurate simulation data for the equation of state of the hard-disk fluid available at present for the above-mentioned density range. Because of that, they are taken here as reference to study the accuracy of several expressions for the HD EOS. The same authors have noted that their results in the region close to the phase transition agree quite accurately with other extensive Monte Carlo simulation data [11, 12], with the exception of the value obtained at reduced density 0.9 . In relation to this, the authors pointed out that this datum could be affected by finite size effects. Therefore, we have not considered here this last value in our calculations.

As mentioned above, a great number of analytical expressions have been proposed for the EOS of HDs, being the values of the virial coefficients and computer simulation data the main sources of information in order to build them. We consider here a number of these equations, which we have classified, in agreement with the desirable requirements mentioned in Section 1, as: a) Accurate analytical expressions; b) EOSs accurately reproducing the virial expansion; c) Theoretically based EOSs; d) Simple EOSs. In the next sections those EOSs are described and two new proposals are included. All of them are compared with the computer simulation data of Kolafa and Rottner [9] for the compressibility factor. 


\section{Accurate analytical expressions for the HD EOS}

We include here those EOSs giving an excellent agreement with computer simulation data for $Z$, which has been possible because of the use of adjustable parameters. Thus, Kolafa and Rottner [9] have fitted their own simulation data to three different analytical expressions depending on the maximum density considered. They are reproduced here for reference.

For $\rho^{*} \leq 0.88$

$$
\begin{gathered}
Z_{K R 1}=1+2 x+1.12801775 x^{2}+0.00181895291 x^{3}-0.0526134737 x^{4}+0.0504951668 x^{5} \\
-0.0325433846 x^{6}+0.0133946531 x^{7}+0.00174265604 x^{8}-0.00944632202 x^{9} \\
+0.00851111768 x^{10}-0.0035963525 x^{11}+0.000577345106 x^{12} \\
-1.06399127 \times 10^{-7} x^{19}
\end{gathered}
$$

For $\rho^{*} \leq 0.89$

$$
\begin{gathered}
Z_{K R 2}=1+2 x+1.12801775 x^{2}+0.00181895291 x^{3}-0.0526134737 x^{4}+0.0504963915 x^{5} \\
-0.0325578581 x^{6}+0.0134816028 x^{7}+0.00129187484 x^{8}-0.00808881628 x^{9} \\
+0.00669011963 x^{10}-0.00250795961 x^{11}+0.000336036442 x^{12} \\
-5.15282664 \times 10^{-9} x^{22}+5.57730095 \times 10^{-23} x^{57}
\end{gathered}
$$

And, for $\rho^{*} \leq 0.90$

$$
Z_{K R 3}=1+2 x+1.12801775 x^{2}+0.00181895291 x^{3}-0.0526134737 x^{4}+0.0504960168 x^{5}
$$




$$
\begin{gathered}
-0.0325537792 x^{6}+0.0134578632 x^{7}+0.00140888182 x^{8}-0.00834273601 x^{9} \\
+0.00694127367 x^{10}-0.00262254723 x^{11}+0.000355746352 x^{12} \\
-5.24672938 \times 10^{-9} x^{22}+5.88054639 \times 10^{-23} x^{57}
\end{gathered}
$$

where $x=\eta /(1-\eta)$. These equations where obtained as the best fitting to the simulation data reported by the same authors [9], in the mentioned ranges, on condition that the virial coefficients $B_{n}$ of Eq. (2) must be reproduced exactly up to $n=5$.

Equations (4) and (5) predict a van der Waals loop for $\rho^{*} \gtrsim 0.89$, whereas Eq. (3) predicts a maximum in the same region. On the other hand, Eq. (5) may be affected by finite-size effects [9] in that region. Therefore, Eq. (4) can be considered as the most accurate EOS in the range were those effects are not present. In particular, the absolute average deviation (AAD) of Eq. (4) with respect to the Kolafa and Rottner simulation data is of only $0.0003 \%$. As Eqs. (3)-(5) are the most accurate available at present for the hard-disk fluid in their respective ranges, there is little more to say with regard to the above-mentioned point 1) of the introduction.

\section{Analytical HD EOSs accurately reproducing the virial expansion}

As mentioned above, Equations (3)-(5) reproduce exactly the virial coefficients $B_{n}$ for $n \leq 5$. They reproduce also the virial coefficients for $6 \leq n \leq 10$ quite accurately, but not exactly. If one wishes to reproduce all the known virial coefficients one has to resort to some kind of approximant. These are functions depending on a number of parameters that are determined from the condition that their series expansion must reproduce the known virial virial coefficients. 
Many kinds of approximants have been proposed in the literature, and practically all of them have been recently compiled [1]. Three of the most popular are the following.

Padé approximants (PA):

$$
Z_{P A}[L / M](\rho)=\frac{1+\sum_{l=1}^{L} p_{l}\left(B_{2} \rho\right)^{l}}{1+\sum_{m=1}^{M} q_{m}\left(B_{2} \rho\right)^{m}},
$$

with the condition that $L+M \leq k-1$, where $k$ is the number of virial coefficients that are to be reproduced exactly.

This kind of approximants have the drawback that we don't know in advance if a particular approximant will be accurate. Neither the use of a greater number of virial coefficients guaranties a greater precision. In general the more symmetric approximants, that is those for which $L \approx M$, are reasonably accurate. Another drawback is that some PA approximants may diverge at inappropriate densities.

Classical HD EOSs based on PA are those of Erpenbeck and Luban (EL) [13] and Sanchez [14], obtained on the basis of six and eight 'exact' virial coefficients, respectively. More recently, Clisby and McCoy (CM) have reported [8] the coefficients of the Padé approximants [4/5] and [5/4], as defined in Eq. (6), reproducing the first ten virial coefficients. They are as follows.

For the PA [5/4]

$$
\begin{gathered}
p_{1}=-0.062894522 \quad ; \quad p_{2}=0.13851476 \quad ; \quad p_{3}=0.0067699403 \quad ; \quad p^{2}=0.0039942056 \quad ; \quad p_{5}=0.00047760798 \quad ; \quad q_{1}=-1.0628945 \quad ; \\
p_{2}=0.41940485 \quad ; \quad q_{3}=-0.11367848 \quad ; \quad q_{4}=0.021846467
\end{gathered}
$$

For the PA $[4 / 5]$ 


$$
\begin{gathered}
p_{1}=0.69939247 \quad ; \quad p_{2}=-0.33033017 \quad ; \quad p_{3}=0.11294457 \quad ; \\
p_{4}=-0.012320562 \quad ; \quad q_{1}=-0.300607528 \quad ; \quad q_{2}=-0.81172709 \quad ; \\
q_{3}=0.62751627 \quad ; \quad q_{4}=-0.17862580 \quad ; \quad q_{5}=0.021359218
\end{gathered}
$$

It is to be noted that the uncertainty in the virial coefficients for $n \geq 5$ gives rise to an uncertainty in the $p_{i}$ and $q_{i}$ coefficients of the order of the coefficients themselves [8].

In Table 1, the AAD of EL, Sanchez and the two CM approximants (CM54 and CM45), with respect to the Kolafa and Rottner simulation data are given for three different density ranges (low and intermediate density, high density, and full density range). The aim is to test to what extent the use of a greater number of 'exact' virial coefficients to construct the PA results in an improved accuracy. As can be seen, the CM45 approximant can be considered as the most accurate of the PAs in the three ranges, with only a minor difference with respect to the CM54 one. Moreover, both PA are more accurate than all the other expressions considered in this work, with the logical exceptions of the KR ones, in the low and intermediate density range $\left(0.4 \leq \rho^{*} \leq 0.75\right)$. As expected, in the high-density range the accuracy is not so good.

The y-expansion [15]

$$
Z=\frac{1}{\eta} \sum_{i=1}^{k} C_{i} y^{i},
$$

where $y \equiv x=\eta /(1-\eta)$ and the coefficients $C_{i}$ are obtained from the virial coefficients through the relationships

$$
C_{i}=\sum_{n=1}^{i}\left(\begin{array}{c}
i-1 \\
n-1
\end{array}\right)(-1)^{i-n} B_{n}^{*}
$$




$$
c_{n}=\sum_{j=0}^{2} \frac{2 !(-1)^{j}}{(2-j) ! j !} B_{n-j}^{*},
$$

in which

$$
Z=\frac{1+\sum_{n=1}^{k} c_{n} \eta^{n}}{(1-\eta)^{2}}
$$

with the condition that $B_{n}^{*}=0$ for $n<0$, and $B_{0}^{*}=1$. As can be seen, in expression (11) the singularity is found at $\eta=1$.

Baus and Colot (BC) reported [16] the coefficients $c_{n}$ up to $n=6$. However, here we have obtained the coefficients up to $n=9$ from the virial coefficients (2). As can be seen in Table 1, the BC EOS gives similar accuracy as the Padé approximants CM at all densities.

To improve the accuracy in the high density region, while reproducing exactly all known virial coefficients, we propose here a new EOS of the form

$$
Z_{S M C 1}=\frac{a_{0}^{2}+\sum_{i=1}^{9} a_{i} \eta^{i}+a_{15} \eta^{15}+a_{20} \eta^{20}+a_{25} \eta^{25}}{\left(a_{0}-\eta\right)^{2}}
$$

where coefficients $a_{i}$ for $1 \leq i \leq 9$ are determined from the condition that the virial coefficients, Eq. (2), must be reproduced, and with $a_{0}, a_{15}, a_{20}$, and $a_{25}$ obtained from the fitting of the simulation data. The results are

$$
a_{0}=0.83908560190 \quad ; \quad a_{1}=-0.27004190916 \quad ; \quad a_{2}=-0.15401569249 \quad ;
$$




$$
\begin{gathered}
a_{3}=-0.25154451976 \quad ; \quad a_{4}=-0.25987073612 \quad ; \quad a_{5}=-0.21843661442 \quad ; \\
a_{6}=-0.16505544817 \quad ; \quad a_{7}=-0.11753138009 \quad ; \quad a_{8}=-0.080835032006 \quad ; \\
a_{9}=-0.048203974532 \quad ; \quad a_{15}=-10.2969062842 \quad ; \quad a_{20}=200.567428335 \quad ; \\
a_{25}=-966.7004411299648
\end{gathered}
$$

As can be seen in Table 1, the new EOS, Eq. (13), gives excellent accuracy in the whole density range, with a maximum deviation of only $0.2 \%\left(\Delta Z=0.021\right.$ at $\left.\rho^{*}=0.89\right)$, and with a significant improvement when compared with other proposals. Obviously, this success is due to the use of a greater number of virial coefficients and of four adjustable coefficients.

We will end this section mentioning the so-called Asymptotic Expansion Method, recently proposed [6]. This is a new kind of approximant, although does not reproduces exactly all the virial coefficients known at present, because it is based on the first few virial coefficients. In particular, Khanpour and Parsafar (KP) proposed four analytical expressions, depending on the number of virial coefficients that are exactly reproduced, as follows:

$$
\begin{gathered}
Z_{K P 11}=\frac{-1+\eta-0.128 \eta^{2}+0.1261 \eta^{3}}{(\eta-1)^{3}} \\
Z_{K P 12}=\frac{-1.331+0.968 \eta-0.2034 \eta^{2}+0.0874 \eta^{3}}{(\eta-1.1)^{3}} \\
Z_{K P 14}=\frac{0.9983-0.0017 \eta+0.1261 \eta^{2}}{(\eta-0.9992)^{2}}
\end{gathered}
$$




$$
Z_{K P 16}=\frac{-1.467+0.9392 \eta-0.2511 \eta^{2}+0.0516 \eta^{3}}{(\eta-1.1363)^{3}}
$$

As can be seen, the poles of several of these equations are different and near to $\eta=1$.

The AADs of the KP EOSs with respect to the Kolafa and Rottner data are compared with those given by other EOSs in Table 1. As can be seen in that Table, only the KP12 EOS, Eq. (16), has a very good accuracy at low and intermediate densities at the same time that at high densities it improves the results obtained from all the other approximants considered here. The obtained AAD with this EOS in the whole density range is slightly lower than the given by the CM45 EOS, but clearly higher than the obtained with the new SMC1 expression proposed here, Eq. (13)

In Figure 1 the results from several equations of state are compared. To enhance the differences, instead of plotting the compressibility factor $Z$ as a function of the reduced density $\rho$ we have plotted the difference $\Delta Z$ between a given equation of state and the computer simulation data of Ref. [9]. For the same reason, only the region $\rho^{*} \geq 0.70$ has been considered. One can see that the Padé approximants [5/4] and [4/5], given by Eq. (6) with coefficients (7) and (8) respectively, deviate from the simulation data for $\rho^{*} \gtrsim 0.82$. In the same Figure one can also see that the KP12 expression, Eq. (16), is slightly less accurate than the CM45 and CM54 ones for $\rho^{*} \leq 0.82$, where it under-predicts the simulation data, but clearly more accurate at higher densities.

\section{Theoretically-based analytical EOSs}

The availability of analytical equations of state for the hard-disk fluid with a sounded theoretical basis is very scarce. It stands out the result provided by the scaled particle theory (SPT) [17]

$$
Z=\frac{1}{(1-\eta)^{2}}
$$


As can be seen in Table 1, this very simple expression gives the highest AAD in the low and intermediate density range. In fact, it provides quite poor accuracy for reduced densities $\rho^{*} \gtrsim 0.5$ (see Fig. 2). Nevertheless, we can consider that this EOS is well balanced, in the sense of that it gives practically the same $\mathrm{AAD}$ at low and intermediate densities as at high densities. In fact, at high densities it gives a lower AAD than that obtained from a significant number of the, analytically more complex, EOSs included in the Table.

Santos et al. [4] proposed an EOS that has not a sound theoretical basis, but which can be considered as semi-theoretical because it does not include any adjustable coefficient. This EOS was obtained by merely imposing an exact reproduction of the second virial coefficient and the existence of a pole singularity at the close-packing fraction. Its analytical expression is:

$$
Z_{S H Y}=\left(1-2 \eta+\frac{2 \eta_{c}-1}{\eta_{c}^{2}} \eta^{2}\right)^{-1}
$$

As can be seen, it is a very simple expression which has another pole at $\eta_{c} /\left(2 \eta_{c}-1\right)$, and which will be referred to as the SHY EOS.

Recently, Mulero et al. [3] have compared the virial coefficients obtained from Eq. (20) with the exact values given in Eq. (2). The obtained results confirm that the SHY EOS provides reasonable estimates of the virial coefficients, performing better than some of the more complex expressions. Santos et al. also noted that the value that Eq. (20) predicts for the seventh virial coefficient is more accurate than that obtained from other expressions which explicitly include up to the sixth coefficient in their construction.

Due to its simplicity, the SHY equation only improves the accuracy of the SPT EOS at densities $\rho^{*} \leq 0.85$, but it gives greater deviations at higher densities (see Fig. 2). In particular, the maximum error found is $13.4 \%$ at the highest density, whereas it is $7 \%$ for the SPT EOS.

Quite recently, Rusanov [7] derived a new family of theoretically-based EOSs on the 
basis of the concept of the exclusion factor (volume or area), which was already used by Boltzmann and van der Waals. As is well known, the exclusion volume is defined as the volume that must be subtracted from the actual volume of a system due to the fact that particles in the system have their own volumes. The family of equations for the HD proposed by Rusanov obey to the general form:

$$
Z^{(n)}=\frac{1}{(1-k \eta)^{n-1}}\left\{1-\frac{2 n k-2 k-4}{(n-1)(n-2) k^{2} \eta}\left[(1-k \eta)^{n-1}-1+(n-1) k \eta\right]\right\}
$$

with $n \geq 3$ being an integer providing different degrees of approximation and $k$ being an adjustable parameter. In particular, for $n=3$, Eq. (21) reduces to

$$
Z^{(3)}=\frac{1-2(k-1) \eta}{(1-k \eta)^{2}}
$$

and for $n=4$

$$
Z^{(4)}=\frac{1-(3 k-2) \eta+k(k-2 / 3) \eta^{2}}{(1-k \eta)^{3}}
$$

Rusanov [7] determines parameter $k$ from the fitting of the simulation data of Ref. [13], so that Eqs. (22) and (23) may be considered as semiempirical in character. In particular, Rusanov proposed the value $k=1.0145$ for $n=3$, founding a maximum deviation of about $1 \%$ with respect to the Erpenbeck and Luban computer simulation data [13]. If the equation is developed until the fourth approximation, i.e. with $n=4$, then $k \approx 0.876677$ and the maximum deviation is reduced to $0.46 \%$. Rusanov noted that the fifth approximation fits a little worse than the formers so that higher degrees should not be considered. However, at that time there were not available simulation results at high densities for comparison.

We have re-calculated the $k$ values by using the Kolafa and Rottner computer simulation data [9], obtaining $k=0.8733984$ for $n=4$. Using this new parameter, the AADs found for $n=4$, Eq. (23), are clearly lower than the ones from the SPT and SHY EOSs in the three density ranges considered (see Table 1), which is obviously because of the use 
of the adjustable coefficient. In particular, in Fig. 2 it is shown that the Rusanov EOS gives clearly better results than the SPT one for $\rho^{*} \leq 0.8$ but it gives similar results at higher densities. When the whole density range is considered, the AAD obtained by using Eq. (23) is even lower than those given by the KP12 EOS, Eq. (16), which is analytically similar.

\section{Simple and accurate EOSs for the hard-disk fluid}

Let us consider now equations of state of the kind mentioned in the point 4) of those quoted in Section 1, that is, equations of state derived on a heuristic basis, reasonably simple and providing satisfactory accuracy as compared with the simulation data and the virial expansion.

The Henderson equation [18]

$$
Z_{H 75}=\frac{1+\eta^{2} / 8}{(1-\eta)^{2}}
$$

can be considered as belonging to this kind of equations. As shown in Table 2, the predicted virial coefficients from this equation are quite reasonable despite its simplicity.

As can be seen in Table 1, its accuracy at low and intermediate densities is even better than that obtained with the simple SPT and SHY EOSs, but the contrary occurs at high densities. Specifically, the accuracy of this EOS is quite satisfactory for $\rho^{*} \lesssim 0.70$ (with $|\Delta Z| \lesssim 0.04$ ) where it gives even slightly better results than the Rusanov EOS, which includes an adjustable coefficient. Moreover, it must be noted that the AADs obtained with the H75 EOS are of similar order as that obtained with the more recent and analytically slightly more complex KP11 and KP14 EOSs in the three density ranges considered.

In order to obtain a better accuracy at high densities, Henderson [19], proposed a further modification by including an additional term, as follows 


$$
Z_{H 77}=\frac{1+\eta^{2} / 8}{(1-\eta)^{2}}-\frac{0.043 \eta^{4}}{(1-\eta)^{3}}
$$

As can be seen in Fig. 2, this new EOS reproduces the simulation data almost exactly for $\rho^{*} \leq 0.83$ (with $|\Delta Z|<0.012$ ). Results in Table 1 show that this EOS clearly improves those of the previous one, with similar results as the more complex KP12 expression (the most accurate of those proposed recently by Khanpour and Parsafar [6]).

Following the same idea, and trying to reproduce better the virial coefficients, a new small correction to Eq. (24) has been proposed by Solana, in the form [2]

$$
Z=\frac{1+\eta^{2} / 8-\eta^{4} / 10}{(1-\eta)^{2}}
$$

As Table 2 shows, this equation provides the same values of the virial coefficients $B_{n}$ as Eq. (24) for $n=3$ and 4; slightly worse for $n=5$ and considerably better for $n>5$, although Eq. (25) provides a still better estimation of the virial coefficients for $n \leq 5$. Moreover, as can be seen in Table 1, Eq. (26) gives slightly higher AADs than Eq. (25) does.

In order to improve the accuracy in the region $0.80 \leq \rho^{*} \leq 0.90$ we can add to the previous expression an 'ad hoc' term with the result

$$
Z=\frac{1+\eta^{2} / 8-\eta^{4} / 10}{(1-\eta)^{2}}-\frac{50000 \eta^{40}}{(1-\eta)^{3}}
$$

This equation, still considerably simple, provides excellent agreement with the simulation data, as seen in Fig. 2 and in Table 1. The AAD at high densities $\left(\rho^{*} \geq 0.8\right)$ is of the same order as that from the analytically more complex Eq. (13), whereas at low and intermediate densities is slightly higher. In any case, it must be taken into account that Eq. (13) includes four coefficients obtained by a fitting procedure, and moreover that it reproduces better the virial coefficients. These results indicate that the new EOS, Eq. (27) can be considered as the most accurate of the simple expressions for the HD EOS in order to reproduce the simulation data available for the compressibility factor. When a 
greater accuracy is needed at densities below 0.8, the Padé approximants CM54 and CM45, and the RVE give better results, with the latter two being the most accurate. When a greater accuracy is needed in the whole density range, then the analytically complex EOS proposed by Kolafa and Rottner, Eqs. (3) to (5) must be used.

\section{Conclusions}

Let us now revise the four points quoted in the first section in light of the preceding results.

1) As far as accuracy is concerned, the most accurate analytical equation of state for the hard-disk fluid available at present for the whole density range $\left(\rho^{*} \leq 0.90\right)$ is Eq. (5), although Eqs. (3) and (4) are the most accurate for $\rho^{*} \leq 0.88$ and $\rho^{*} \leq 0.89$, respectively.

2) Regarding equations of state reproducing exactly the first ten virial coefficients, the approximants analyzed in section 2, do not provide enough accuracy in the high density region, and the very recently proposed Asymptotic Expansion Method neither reproduces exactly all the known virial coefficients, so that there is room for improvements. This has been achieved introducing a new approximant, Eq. (13) with four adjustable parameters. This lead to an equation of state that not only reproduce exactly all $B_{n}$ for $n \leq 10$, but also is very accurate for the whole density range.

3) The scarce theoretically-based equations of state available do not provide enough accuracy in reproducing the existing simulation data nor the virial coefficients, so that this field is open to future developments.

4) Finally, the simple equations of state derived on an heuristic basis available in the literature are also lacking of enough accuracy in predicting these properties. In order to attempt to remedy this situation, we propose Eq. (27) that is quite simple and extremely accurate for the whole density range. Moreover, it gives quite good estimates of the known virial coefficients.

From the preceding comments, it is to be expected little progress in the 
future concerning the points 1), 2), and 4). Therefore, it would be desirably to focus future efforts mainly within the context of point 3 ). Theoretical achievements for two-dimensional fluids can be often extended to their three-dimensional counterparts. In particular, the availability of analytical expressions for the equation of state and the radial distribution function for the hard-sphere fluid accurate up to the high density region is of crucial importance within the context of perturbation theories. The latter are widely applied to a great variety of fluids and are the subject of continuous advances. Two of the most recent outstanding advances in this field are the proposal of a general perturbation theory for the thermodynamic properties of fluids based on a coupling parameter expansion around a reference hard-sphere fluid proposed by Zhou up to third order [20] and subsequently extended by the same author up to fifth order [21], and the development of a perturbation theory for systems with short-ranged interactions [22].

The present work has been partially supported by the Spanish Dirección General de Investigación (DGI) under grant FIS2006-02794 FEDER (A.M. and I.C.) 


\section{References}

[1] A. Mulero, Ed. "Theory and Simulation of Hard-Sphere Fluids and Related Systems", Lecture Note Physics 753. Springer (2008).

[2] J.R. Solana, in: La Investigación del Grupo Especializado de Termodinámica de las R.S.E.F. y R.S.E.Q., Año 2006, ed. J.M. Ortiz de Zárate, M. Khayet (R.S.E.F., Madrid) (in Spanish) 306 (2006).

[3] A. Mulero, C.A. Galán, M.I. Parra, and F. Cuadros, in: "Theory and Simulation of Hard-Sphere Fluids and Related Systems", Ed. A. Mulero, Lecture Note Physics 753, Chapter 3. Springer (2008)

[4] A. Santos, M.L. Haro, and S.B. Yuste, J. Chem. Phys. 103, 4622 (1995)

[5] A. Mulero, F. Cuadros, and C.A. Galán, J. Chem. Phys. 107, 6887 (1997)

[6] M. Khanpour and G.A. Parsafar, Fluid Phase Eq. 262, 157 (2007)

[7] A. I. Rusanov, Rus. Chem. Rev. 74, 117 (2005).

[8] N. Clisby and B.M. McCoy, J. Stat. Phys. 122, 15 (2006). See also N. Clisby and B.M. McCoy, arXiv:cond-mat/0503525 (2005), available at the URL http://arxiv.org/archive/cond-mat.

[9] J. Kolafa and M. Rottner, Mol. Phys. 104, 3434 (2006).

[10] A. Malijevsky and J. Kolafa, in "Introduction to the Thermodynamics of Hard Spheres and Related Systems", Ed. A. Mulero, Lecture Note Physics 753, Chapter 2. Springer (2008).

[11] A. Jaster, Phys. Rev. E 59, 2594 (1999).

[12] C.H. Mak, Phys. Rev. E 73, 65104 (2006).

[13] J.J. Erpenbeck and M. Luban, Phys. Rev. A 32, 2920 (1985). 
[14] I.C. Sanchez, J. Chem. Phys. 101, 7003 (1994)

[15] B. Barboy and M. Gelbart, J. Chem. Phys. 71, 3053 (1979).

[16] M. Baus and J.L. Colot, Phys. Rev. A 36, 3912 (1987).

[17] E. Helfand, H.L. Frisch, and J. L. Lebowitz, J. Chem. Phys. 34, 1037 (1961).

[18] D. Henderson, Mol. Phys. 30, 971 (1975).

[19] D. Henderson, Mol. Phys. 34, 301 (1977)

[20] S. Zhou, Phys. Rev. E 74, 031119 (2006); ibid., J. Chem. Phys. 125, 144518 (2006); ibid. J. Phys. Chem. B 111, 10736 (2007).

[21] S. Zhou, Phys. Rev. E 77, 041110 (2008).

[22] P. Sillrén and J.-P. Hansen, Mol. Phys. 105, 1803 (2007). 


\section{Tables}

Table 1: Average absolute deviations, AAD (\%), between the results obtained from the EOSs and computer simulation data of reference [9] for HD systems. Different density ranges $\rho^{*}$ are considered.

\begin{tabular}{lccc}
\hline & \multicolumn{3}{c}{ Density range } \\
EOS & $0.4-0.75$ & $0.8-0.89$ & $0.4-0.89$ \\
\hline EL, Eq. (6) [13] & 0.005 & 3.27 & 1.64 \\
Sanchez, Eq. (6) [14] & 0.076 & 4.45 & 2.26 \\
CM54, Eq. (6) [8] & 0.003 & 3.22 & 1.61 \\
CM45, Eq. (6) [8] & 0.002 & 3.11 & 1.56 \\
BC, Eq. (11)† [16] & 0.002 & 3.16 & 1.58 \\
SMC1, Eq. (13) (this work) & 0.040 & 0.087 & 0.064 \\
KP11, Eq. (15) [6] & 0.486 & 5.58 & 3.04 \\
KP12, Eq. (16) [6] & 0.060 & 2.17 & 1.12 \\
KP14, Eq. (17) [6] & 0.468 & 5.56 & 3.01 \\
KP16, Eq. (18) [6] & 0.371 & 2.05 & 1.21 \\
SPT, Eq. (19) [17] & 2.200 & 2.69 & 2.45 \\
SHY, Eq. (20) [4] & 1.299 & 4.36 & 2.83 \\
RUS, Eq. (23) [7] & 0.171 & 1.84 & 1.01 \\
H75, Eq. (24) [18] & 0.385 & 5.27 & 2.83 \\
H77, Eq. (25) [19] & 0.068 & 2.64 & 1.36 \\
SOL, Eq. (26) [2] & 0.117 & 3.25 & 1.68 \\
SMC2, Eq. (27) (this work) & 0.118 & 0.086 & 0.102 \\
\hline
\end{tabular}

$\dagger$ We have used the virial coefficients (2) up to $n=10$ 
Table 2: Virial coefficients of the hard-disk fluid in the expansion of the compressibility factor in power series of the packing fraction.

\begin{tabular}{ccccc}
\hline \multirow{2}{*}{$n$} & \multicolumn{4}{c}{$B_{n}^{*}=B_{b} / v_{m}^{n-1}$} \\
\cline { 2 - 4 } & exact & Eq. (24) & Eq. (25) & Eq. (26) \\
\hline 2 & 2 & 2 & 2 & 2 \\
3 & 3.1280 & 3.125 & 3.125 & 3.125 \\
4 & 4.2579 & 4.250 & 4.250 & 4.250 \\
5 & 5.3369 & 5.375 & 5.332 & 5.275 \\
6 & 6.3630 & 6.500 & 6.371 & 6.300 \\
7 & 7.3519 & 7.625 & 7.367 & 7.325 \\
8 & 8.3191 & 8.750 & 8.32 & 8.35 \\
9 & 9.2721 & 9.875 & 9.23 & 9.375 \\
10 & 10.2163 & 11.000 & 10.097 & 10.400 \\
\hline
\end{tabular}




\section{Figure captions}

Figure 1: Differences $\Delta Z=Z_{E O S}-Z$ between different equations of state and the computer simulation data [9] as a function of the reduced density $\rho^{*}$ in the high density region.

Figure 2: As in Fig. 1 for other set of equation of state in the medium and high density regions. 


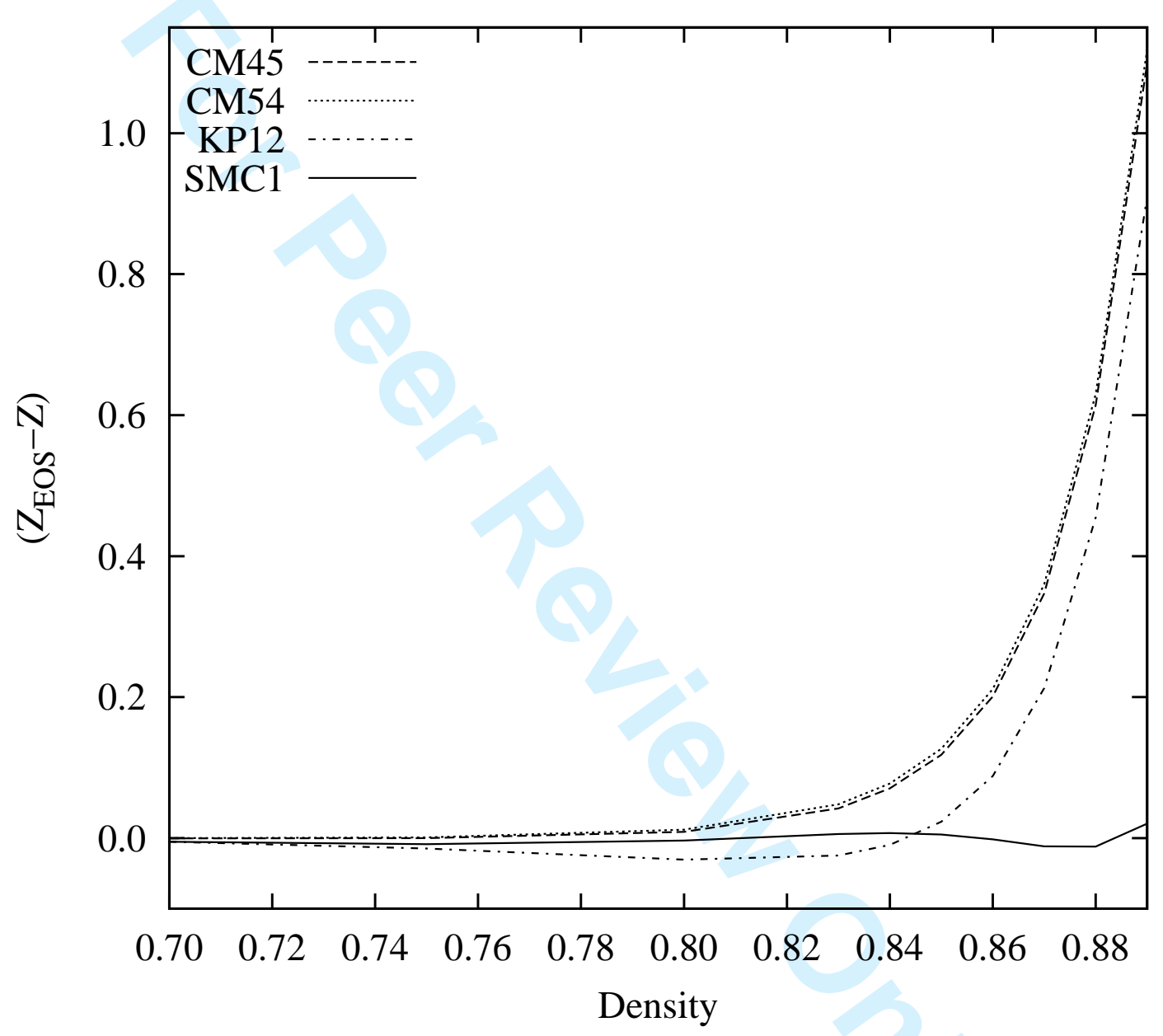

Figure 1: 


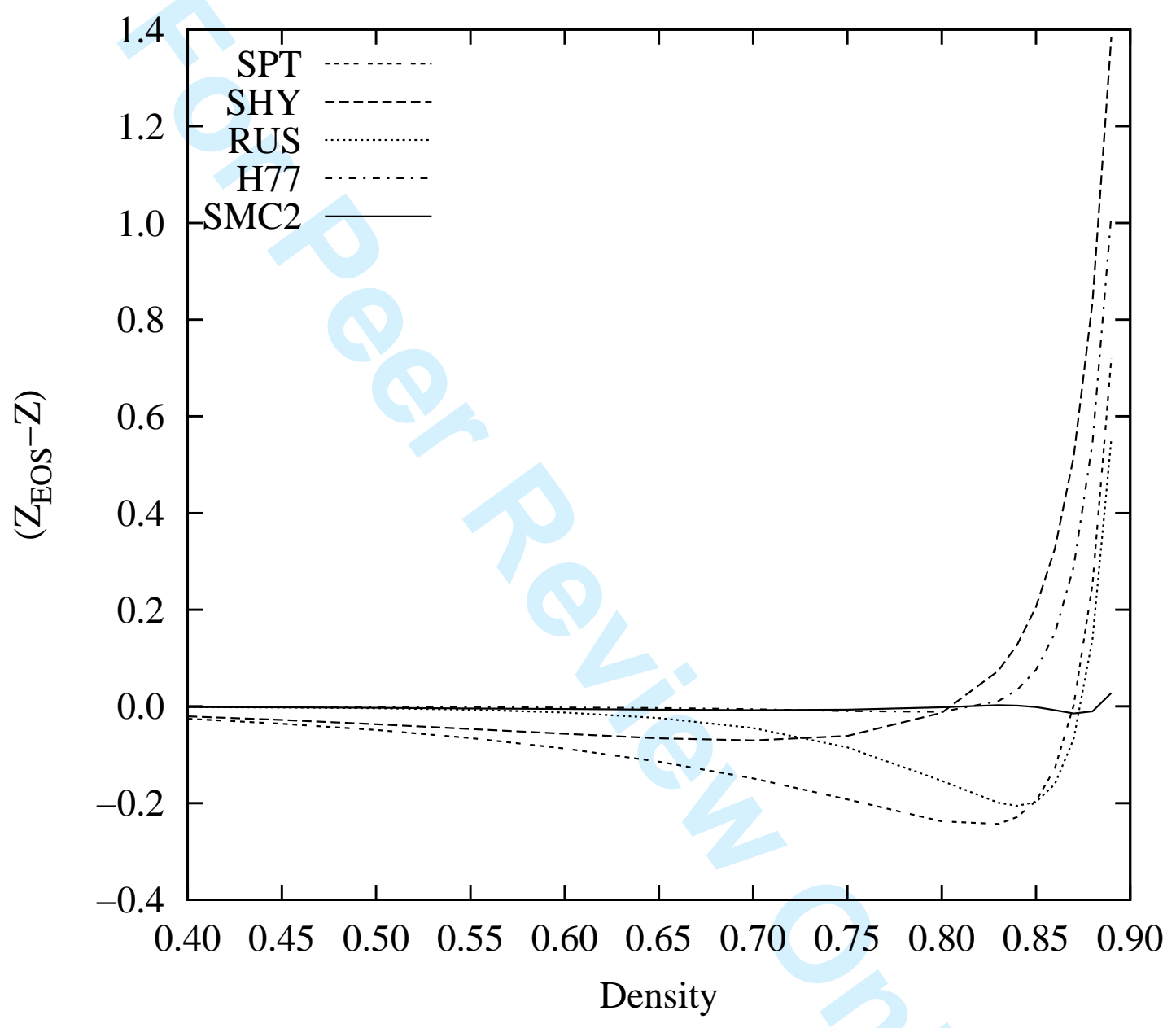

Figure 2: 


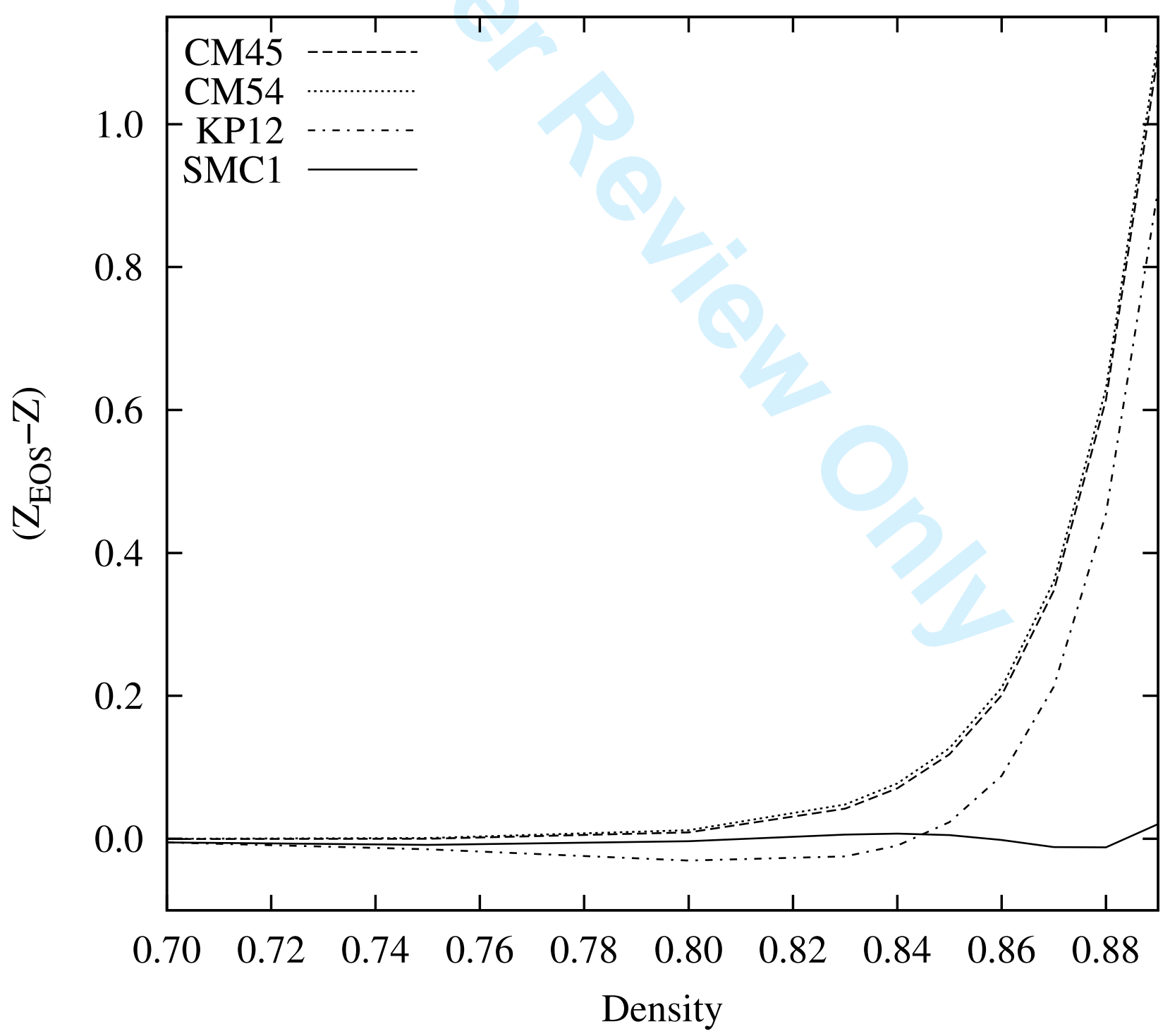

URL: http://mc.manuscriptcentral.com/tandf/tmph 


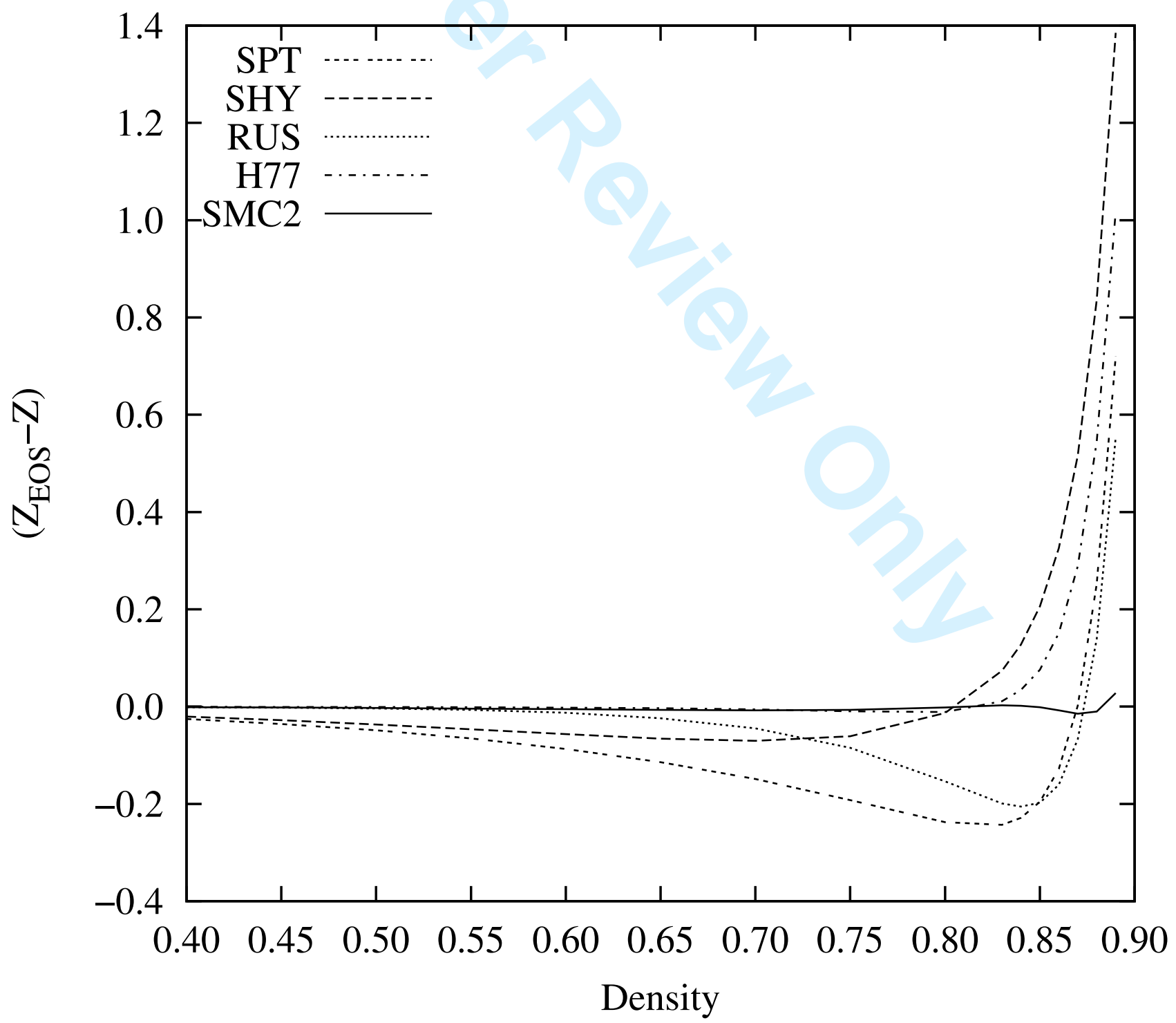

URL: http://mc.manuscriptcentral.com/tandf/tmph 